\title{
Machine learning algorithm for improving performance on 3 AQ-screening classification
}

\author{
Taftazani Ghazi Pratama*, Rudy Hartanto, Noor Akhmad Setiawan \\ Department of Electrical Engineering and Information Technology, Universitas Gadjah Mada, Jl. Grafika No.2, Yogyakarta 55281, Indonesia
}

Article history:

Received: 24 May 2019 / Received in revised form: 24 July 2019 / Accepted: 18 September 2019

\begin{abstract}
Autism Spectrum Disorder (ASD) classification using machine learning can help parents, caregivers, psychiatrists, and patients to obtain the results of early detection of ASD. In this study, the dataset used is the autism-spectrum quotient for child, adolescent and adult, namely AQchild, AQ-adolescent, AQ-adult. This study aims to improve the sensitivity and specificity of previous studies so that the classification results of ASD are better characterized by the reduced misclassification. The algorithm applied in this study: support vector machine (SVM), random forest (RF), artificial neural network (ANN). The evaluation results using 10-fold cross validation showed that RF succeeded in producing higher adult AQ sensitivity, which was $87.89 \%$. The increase in the specificity level of AQ-Adolescents is better produced using an SVM of $86.33 \%$.
\end{abstract}

Keywords: Classification, ASD, machine learning, sensitivity, specificity, SVM, RF, ANN

\section{Introduction}

ASD (Autism Spectrum Disorder) is a neurological development disorder in individuals that can be identified through characteristics such as repetitive behaviour, limited social interaction and communication [1]. The causes of the emergence of ASD are unknown and clear [2]. Some factors that influence the emergence of ASD are: genetic, environmental, and immunologists of an individual. Children aged 2-9 years assessment of diagnosis involves professional experts [3]. According to the Centers for Disease Control and Prevention (CDC), an individual can be diagnosed with ASD by going through two stages: 1. Initial screening / detection 2 . Interview with psychiatrists with children or parents and observing behaviour by pediatricians and neurologists [4].

At this time the instruments used for screening ASD are Autism Behavior Checklist (ABC), Autism Spectrum Quotient (AQ), Child Behavior Checklist (CBCL), and many others [57]. Inside the instrument there are many items that represent questions related to the characteristics of ASD. The use of the instrument is carried out by parents, teachers, caregivers, and patients themselves. However, with many answers that must be filled, it makes the time taken longer and inefficient. Therefore, fewer items are needed in order to speed up the ASD screening process. The solution provided by C. Allison et al. [7] produces screening instruments with fewer items. For example, AQ that is applied at the age of toddler to adult from the beginning has a number of items of 50 and then decreases to 10 items. The items in the ASD screening instrument are

* Corresponding author.

Email: taftazani.ghazi.p@mail.ugm.ac.id given to the patient to be filled in or answered in order to find out the condition of the patient regarding the characteristics that exist whether they meet the ASD criteria or not. The ASD classification in particular using Autism-Quotient has guidelines provided by the National Institute for Health and Care Excellence (NICE) [8,9] to assess each item during the screening process. In addition to the existing guidelines, ASD classification can also be perform using machine learning. Machine learning can classify individuals as indicated by ASD by learning from previous data. Previous studies related to ASD classification using the AQ-Screening methods (AQchild, AQ-adolescent, AQ-adult) have been carried out by Thabtah et al. [10] by means of a feature selection method: Variable analysis (Va). The feature selection results from $\mathrm{Va}$ obtained the following subset of features: AQ-child obtain 8 features, AQ-adolescent obtain 8 features, and AQ-adult obtain 6 features. The subsets of features obtained along with the existing instances are then classified using the classification algorithm: C4.5 and Ripper.

However, the classification results using C4.5 and Ripper showed a misclassification that caused a low sensitivity level on AQ-adult and a low specificity level on AQ-Adolescent. Hence, it leads to poor performance of the prediction results of patients who are being identified as an ASD. With this in mind, several studies has been conducted to increase the sensitivity and specificity by proposing better classification characterized algorithms to reduce the misclassification. Some of them are successfully producing higher sensitivity and specificity algorithms, especially algorithms that related to ASD classification and other medical fields [11-15]. Previous studies conducted by Komisicky et al. [11], autistic detection using SVM, Naïve Bayes, decision tree variants, RF. Some of these algorithms, SVM and RF can produce higher sensitivity 
and specificity than other algorithms. The dataset used is the Autism Diagnostic Observation Schedule (ADOS) in module 2 and module 3 obtained from 5 autism organizations, namely: Autism Consortium (AC), Autism Genetic Resource Exchange (AGRE), National Autism Research Database (NDAR), Simons Simplex Collection (SSC), Simons Variance in Individual Projects (SSVIP).

Another study conducted by Maenner et al. [12] using RF for ASD classification through words or sentences written with supervision of various clinical and school supervision . A textbased dataset obtained from Georgia ADDM (The Autism and Developmental Disabilities Monitoring). In addition to ASD classification through data, ASD classification can also be done through brain image as done by Zhang et al. [13] using SVM. Image-based datasets that describe brain images was obtained from the Autism Research Center through Children's Hospital of Philadelphia.

In other medical fields, in the study [14] which classified chronic kidney disease using SVM and ANN. The higher levels of sensitivity are generated by ANN than SVM. The dataset is obtained from Apollo Hospitals, Tamilnadu, India.

In research by [15], the authors compared to machine learning algorithms: RF, Logistic Regression (LR), ANN (MLP) for predictions of fatty liver disease. The results of optimal sensitivity and specificity is generated by RF. While ANN is third after LR. However, these previous studies did not yet apply their algorithms on ASD datasets in this study. So, this study will use SVM, RF, and ANN to acquire a higher sensitivity and specificity algorithms using the same algorithms as the [11-15]. The aim of this study is to increase the level of sensitivity and specificity in previous studies [10] for ASD classification on 3 AQ-Screening (AQ-Child, AQAdolescent, and AQ-Adult).

\section{Materials and Method}

\subsection{Dataset}

The dataset used in this study was obtained from the UCI Machine Learning Repository [16-18] consisting of 3 agerelated ASD screening datasets from children to adults. AQChildren, AQ-Adolescent, AQ-Adult have the same number of features or attributes: 21 . The number of instances of the 3 datasets is different because there are different proportions between ASD and non-ASD classes. The number of instances contained in the three datasets amounts to the following: 292, 104, and 704.

Features and Description are shown in Table 1. In Table 1, the values contained in A1-A10 attributes in each dataset are coded with 0 or 1 depending on the answers given by the participant during the ASD screening process.

In Table 2, 10 questions in one of the AQ-Screening Methods are presented, namely AQ-10 Child. Each question represents the categories in ASD screening. Giving a value of 0 or 1 for each dataset varies. For AQ-Child on questions A1, A5, A7, A10 coded with value 1 if the participant chooses answer definitely or slightly agree and value 0 if the participant chooses answer definitely or slightly disagree. In questions A2, A3, A4, A6, A8, A9 is coded with a value of 1 if the participant chooses the answer definitely or slightly disagree. While the value of 0 if the participant chooses the answer definitely or slightly agree. For AQ-Adolescent, the value 1 is set for questions $\mathrm{A} 1, \mathrm{~A} 5, \mathrm{~A} 8$, and $\mathrm{A} 10$ if the participant answers definitely or slightly agree.
While the value of 0 is determined if the participant answers otherwise. On the remaining questions, if the participant answers definitely or slightly disagree, he will get a value of 1 and otherwise will get a value of 0 . For the last type of AQ (AQ-Adult), the value 1 is for questions A1, A7, A8, and $\mathrm{A} 10$ if the participant answers definitely or slightly agrees. Conversely, if the participant answers definitely or slightly disagree, the value 0 is set. Whereas questions A2, A3, A4, A5, A6, and A9 will be assigned a value of 1 if the participant answers definitely or slightly disagree, the value of 0 will be determined if the participant answers otherwise.

Table 1. AQ-Screening Dataset

\begin{tabular}{|c|c|c|}
\hline Feature & Type & Description \\
\hline Age & Number & Participant age \\
\hline Gender & String & Male or female \\
\hline Ethnic & String & Ethnic participant \\
\hline $\begin{array}{l}\text { Born with } \\
\text { jaundice }\end{array}$ & $\begin{array}{l}\text { Boolean } \\
\text { (yes or no) }\end{array}$ & Does jaundice appear at birth \\
\hline $\begin{array}{l}\text { A native of } \\
\text { autistic family }\end{array}$ & $\begin{array}{l}\text { Boolean } \\
\text { (yes or no) }\end{array}$ & $\begin{array}{l}\text { Wheteher families have history of } \\
\text { autism }\end{array}$ \\
\hline $\begin{array}{l}\text { Who does the } \\
\text { screening }\end{array}$ & String & $\begin{array}{l}\text { Parent, self, caregiver, medical staff, } \\
\text { clinician, etc }\end{array}$ \\
\hline Country & String & List of participant countries \\
\hline $\begin{array}{l}\text { Have already } \\
\text { screened through } \\
\text { the application }\end{array}$ & $\begin{array}{l}\text { Boolean } \\
\text { (yes or no) }\end{array}$ & $\begin{array}{l}\text { Has the participant ever screened } \\
\text { through the previous application? }\end{array}$ \\
\hline Description of age & String & $\begin{array}{l}\text { The age range of children, } \\
\text { adolescents and adults }\end{array}$ \\
\hline $\begin{array}{l}\text { First to Tenth } \\
\text { question answer } \\
\text { (A1-A10) }\end{array}$ & $\begin{array}{l}\text { Binary }(0, \\
1)\end{array}$ & The question can be seen on Table 2 \\
\hline Seening Score & Integer & $\begin{array}{l}\text { The final result of screening through } \\
\text { the app with interval rate } 1-10\end{array}$ \\
\hline
\end{tabular}

Table 2. Detail Questions on AQ-10 Child

\begin{tabular}{|c|c|}
\hline Feature & The Questions on AQ-10 Child \\
\hline A1 & He or she often noticed small voices while others didn't \\
\hline A2 & $\begin{array}{l}\text { He or she usually concentrates on the whole picture rather } \\
\text { than the small details }\end{array}$ \\
\hline A3 & $\begin{array}{l}\text { In social groups, conversations of different people can easily } \\
\text { be tracked }\end{array}$ \\
\hline A4 & $\begin{array}{l}\text { He or she felt it was easy to go back and forth between } \\
\text { various activities }\end{array}$ \\
\hline A5 & $\begin{array}{l}\text { He or she doesn't know how to maintain conversations with } \\
\text { friends }\end{array}$ \\
\hline A6 & He or she is good at social chatting \\
\hline A7 & $\begin{array}{l}\text { When he or she reads a story, he or she finds it difficult to } \\
\text { know the character's intentions or feelings }\end{array}$ \\
\hline A8 & $\begin{array}{l}\text { When he or she was in preschool, playing a game } \\
\text { pretending he or she always enjoys }\end{array}$ \\
\hline A9 & $\begin{array}{l}\text { He or she found it easy to know what someone was thinking } \\
\text { or feeling just by looking at their faces }\end{array}$ \\
\hline A10 & He or she found it difficult to make new friends \\
\hline
\end{tabular}

\subsection{Information Gain}

Information Gain is a measure used to calculate how much information is obtained from a feature on a class [19]. The 
formula for Information Gain can be seen in Equation (1).

$$
\operatorname{Gain}(X, A)=E(X)-\sum_{i=0}^{n} \frac{|X i|}{|X|} * E(X i)
$$

where $X$ is the set of cases, $E$ is Entropy, $n$ is the number of partitions of attribute $A,|X i|$ is the number of cases of partition $I$, and $|X|$ is the number of cases in $X$.

\subsection{Chi-Square}

Chi-Square is a method for calculating correlations between 2 variables. Non-class variables with class variables where the data types of the two variables are nominal [20]. The formula for Chi-Square can be seen in Equation (2).

$$
\text { Chi }- \text { Square }=\frac{N \cdot(A D-B C)^{2}}{(A+B) x(C+D) x(A+C) x(B+D)}
$$

where $N$ is the number of instances in training data, $A$ is the frequency of non-class variables with class variables, $B$ is the frequency of non-class variables without class variables, $C$ is the frequency of class variables without non-class variables, and $D$ is the frequency of unbound instances on non-class variables and class variables.

\subsection{Variable analysis (Va)}

Variable analysis is a filter-based feature selection method made by Thabtah et al. [10] by combining Information Gain and Chi-Square to get fewer features in the dataset. $\mathrm{Va}$ produces a score for selected features that are optimal by not decrease the performance level of the classification model badly.

$V a$ combines the $I G$ and Chi-Square scores with the following steps: 1 . Calculate the selected attribute or feature score using IG and Chi-Square; 2. Normalize the score of each feature from the calculation of IG and Chi-Square respectively; 3 . Calculate the magnitude of score from the previous normalization process by combining the two scores of each attribute of the $I G$ calculation and Chi-Square

The result of $V a$ is a new vector based on the $I G$ and ChiSquare score each feature and calculates the magnitude of the vector which is also called $M$ score. $M \_S c o r e$ in $V a$ is using as a strong measure in choosing optimal features. Mathematical formulations are shown in Equations (3) - (6).

$$
\overline{C h l S Q v}=\frac{\text { ChiSQv }}{\text { ChiSQmax }}
$$

where $\overline{C h l S Q v}$ is the result of normalization of ChiSQ scores for each attribute with the maximum $C H i S Q$ score of features, $C h i S Q v$ is the ChiSQ score generated on each feature, and ChiSQmax is the maximum ChiSQ score.

$$
\overline{I G v}=\frac{I G v}{I G \max }
$$

where $\overline{I G v}$ is the result of normalization of IG scores for each attribute variable with maximum IG score of features, IG is the Information Gain score generated on each feature, and IGmax is the maximum IG score.

The score vector of each feature(v) can be defined to be

$$
S V y=\left(\frac{I G v}{C h i S Q v}\right)
$$

The magnitude of the score vector is calculated by summing ChiSQv and $I G v$ from each attribute and then squaring it then rooting it. Formula for vector size is shown in Equation (5).

$$
|S V y|=\sqrt{(I G v)^{2}+(C h i S Q v)^{2}}
$$

\subsection{Support Vector Machine (SVM)}

SVM is a machine learning algorithm made by Vapnik in 1995 which is used for classification problems and along with the development of this classifier can solve regression problems. SVM has the task of separating 2 classes linearly and non-linearly. The dividing line in SVM is called Hyperplane.

Hyperplane can be said to be the best separator between two classes by measuring the hyperplane margin and looking for the maximum point. Margin is the distance between the hyperplane and the closest data point in each class. Support vector is a line that determines the closest data point.

SVM has a way to handle classification for data that cannot be separated linearly. This method is called the kernel trick. The types of kernels that are often used when using SVM include: linear, polynomial, Radial Basis Function (RBF), sigmoid [21].

Table 3. The Types of Kernel

\begin{tabular}{ll}
\hline Kernel Function & Expression \\
\hline Linear & $K(x, y)=x \cdot y$ \\
Polynomial & $K(x, y)=(x \cdot y+1)^{d}$ \\
RBF & $K(x, y)=\exp \left(-g \cdot|u-v|^{2}\right)$ \\
Sigmoid & $K(x, y)=\tan (x \cdot y+b)$ \\
\hline
\end{tabular}

\subsection{Random Forest (RF)}

Random Forest is a machine learning algorithm developed by Breiman for classification and regression problems. Random Forest has the task of making multiple random trees based on training data and randomly selecting the root node with the same distribution per sample. Prediction results were chosen based on a majority vote.

The Majority votes are calculation proportion of the number of classes in the model which decision trees are counted more. Calculation most classes are chosen to predict a case. If a majority vote $>0$ then the result of the prediction will result in higher accuracy. If the majority vote $<0$ then the result predictions will have a high error rate [22].

\subsection{Artificial Neural Network (ANN)}

ANN is a machine learning algorithm that adopted the human brain system in producing information. ANN is often referred to as a neuron which consists of several elements such as input layer, hidden layer, weight, and output layer. Input layer functions as a signal receiver, hidden layer to hide the desired prediction, weight functioned as a liaison between 
the input layer and the hidden layer, the output layer functioned as another nerve.

\subsection{Methodology}

Fig.1 shows the paths carried out in this study. The dataset used in this study is related to ASD screening obtained from the UCI Machine Learning Repository which consists of 3 datasets namely: AQ-Screening Child, AQ-Screening Adolescent, AQ-Screening Adult [16-18].

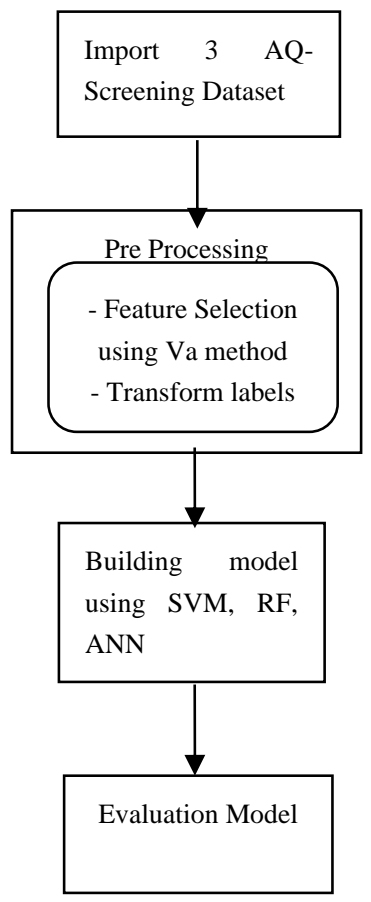

Fig. 1. Research Stages

Before classifying ASD using a machine learning algorithm, all features in the dataset are selected using the Va method to obtain a subset of relevant features in the ASD screening process and transform non numerical labels to numerical labels. After the feature subset is obtained and label have been transformed, the dataset is separated into training data and data testing using 10-fold cross validation.

10 -fold cross validation is one of the validation technique that functions as divider the dataset into 10 parts. In every 1 part, there are $90 \%$ training data and $10 \%$ testing. The distribution of training data and testing is done randomly 10 times. The process of modelling is done by using training data by involving machine learning algorithms such as SVM, RF, and ANN.

Then the model is tested on testing data that is not involved in the training process. The results of the performance of each machine learning algorithm are compared and measured using performance metrics such as sensitivity and specificity.

\section{Results and Discussion}

In this study, the datasets used were: AQ-Child Dataset, AQ-Adolescent Dataset, and AQ-Adult Dataset with 10 features each. There are 2 stages carried out in this study, namely: First, Pre-processing is done in WEKA version 3.8.1 [23] where Va method has been implemented in it. To get fewer features from the three datasets, Va method involves the process of ranking attributes and threshold settings. The threshold is set to $50 \%$ to be able to automatically determine the relevant features to the selection process.

Each feature of all three datasets that have values below $50 \%$ will be deleted or omitted. The results of the subset of features in the three datasets obtained by $\mathrm{Va}$ are 8 features on the AQ-Child dataset, 8 features on the AQ-Adolescent dataset, and 6 features on the AQ-Adult dataset. Before classifying, labels are transformed from non-numerical to numerical using label Encoder that provided by Scikit learn [24]. Second, the ASD classification process uses machine learning algorithms, among others: SVM, RF, and ANN.

Whereas SVM with linear, RF, and ANN based Multi Layer Perceptron (MLP) are implemented using Scikit learn [24]. MLP used in this study is the default parameter provided by the scikit learn [24]. The details are as follows: the hidden layer is 100, the activation function using 'Relu', the optimization of weight using 'Adam', the init learning rate is set to 0.001 . The general size used to see the classification results (ASD, No ASD), namely: Confusion matrix shown in Table 4. From Confusion Matrix, there are other measurements such as Sensitivity (Equation 6), Specificity (Equation 7).

Sensitivity was used to determine the proportion of instance tests that were classified correctly in positive cases of ASD. Specificity is used to find out the proportion of instance tests that are classified correctly in negative ASD cases. To evaluate the sensitivity and specificity of the models made, 10fold cross validation is applied. The evaluation results with 10fold cross validation in the form of an average value of all models made from 1 fold to 10 fold.

The focus of this research is only to improve the performance of previous studies [10] specifically for the level of sensitivity and specificity. Accuracy is not a measure of performance assessment in this study because of the problems that occurred in previous studies, namely: the existence of quite a number of misclassifications carried out by the previous classification algorithm [10] causing a low level of sensitivity and specificity.

Table 4 shows the component of data classification assessment which is explained as follows:

1. True positive shows the number of instances correctly identified in positive ASD cases.

2. False positives indicate the number of instances incorrectly identified in a positive ASD cases.

3. True Negative indicates the number of instances correctly identified in negative ASD cases.

4. False Negative indicates the number of instances incorrectly identified in negative ASD cases.

$$
\begin{aligned}
& \text { Sensitivity }=\frac{T P}{T P+F N} \\
& \text { Specificity }=\frac{T N}{T N+F P}
\end{aligned}
$$

Table 4. Confusion Matrix for ASD Classification

\begin{tabular}{lcl}
\hline & Predicted ASD & Predicted No ASD \\
\hline Actual ASD & True Positive (TP) & False Negative (FN) \\
Actual No ASD & False Positive (FP) & True Negative (TN) \\
\hline
\end{tabular}

Table 5 shows the results of evaluating the average sensitivity using 10-fold cross validation from each classifier in the ASD classification on the three datasets. In the previous study [10], the lowest level of sensitivity with the C4.5 
classifier occurred at Adult AQ: $80.95 \%$. This happens because of ASD misclassification. Of the 189 instances which in the case of real positive ASD, 36 instances were classified incorrectly negatively ASD (FN). 36 instances in real cases should be positive ASD. In this study, classifiers who succeeded in increasing the sensitivity level were higher at AQ-Adult namely: RF with a sensitivity of $87.89 \%$. This result was obtained because it successfully reduced ASD misclassification.

Table 5. The result of classifier sensitivity

\begin{tabular}{llllll}
\hline \multirow{2}{*}{ Dataset } & \multicolumn{5}{c}{ Classifier } \\
\cline { 2 - 6 } & $\begin{array}{l}\text { C4.5 } \\
{[10]}\end{array}$ & $\begin{array}{l}\text { Ripper } \\
{[\mathbf{1 0}]}\end{array}$ & $\begin{array}{l}\text { SVM } \\
\text { (This }\end{array}$ & $\begin{array}{l}\text { RF } \\
\text { (This }\end{array}$ & $\begin{array}{l}\text { ANN } \\
\text { (This study) } \\
\end{array}$ \\
& & & study) & study) & \\
\hline AQ-Child & 85.82 & 82.98 & $\mathbf{9 5 . 6 1}$ & 91.21 & 92.78 \\
AQ-Adolescent & 90.48 & 87.30 & 92.74 & 92.74 & $\mathbf{9 7 . 3 2}$ \\
AQ-Adult & 80.95 & 82.54 & 86.81 & $\mathbf{8 7 . 8 9}$ & 83.14 \\
\hline
\end{tabular}

Of the 189 instances which in the case of real positive ASD, only 24 instances were incorrectly classified as negative ASD (FN). In the overall comparison of five classifiers in the classification of the three datasets, the classifier that produced the highest level of sensitivity was: ANN of $97.32 \%$ in the AQ-Adolescent dataset.

Table 6 shows the results of evaluating the average specificity using 10-fold cross validation from each classifier in the ASD classification on the three datasets. In the previous study, the lowest specificity level with the C4.5 classifier occurred at AQ-Adolescent: $78.00 \%$. This happens because of ASD misclassification. Of the 41 instances which in real negative ASD cases, 9 instances were classified incorrectly positive ASD (FP). 9 instances in real cases should be negative ASD.

Table 6 . The result of classifier specificity

\begin{tabular}{llllll}
\hline \multirow{2}{*}{ Dataset } & \multicolumn{5}{c}{ Classifier } \\
\cline { 2 - 6 } & $\mathbf{C 4 . 5}$ & Ripper & SVM & RF & ANN \\
& {$[\mathbf{1 0}]$} & {$[\mathbf{1 0}]$} & (This study) & $\begin{array}{l}\text { (This } \\
\text { study) }\end{array}$ & (This study) \\
\hline AQ-Child & 83.40 & 80.10 & $\mathbf{9 1 . 8 5}$ & 91.12 & 91.67 \\
AQ-Adolescent & 78.00 & 80.50 & $\mathbf{8 6 . 3 3}$ & 83.81 & 73.89 \\
AQ-Adult & 92.00 & 91.18 & 92.41 & 92.53 & $\mathbf{9 4 . 2 7}$ \\
\hline
\end{tabular}

In this study, the classifier that succeeded in increasing the level of specificity was higher at AQ-Adolescent, namely: SVM with a specificity of $86.33 \%$. This result was obtained because it successfully reduced ASD misclassification. Of the 41 instances which in real negative ASD cases, only 6 instances were classified incorrectly positive ASD (FP). In the overall comparison of five classifiers in the classification of the three datasets, the classifier which produced the highest level of sensitivity was: ANN of $94.27 \%$ on the AQ-Adult Dataset.

Based on the results of this study, there are no specific factors or tuning parameters that have an impact on the performance results of the ASD classification model better than previous studies [10]. We only use the default parameters for ANN and Random Forest in improving the ASD classification performance. Whereas SVM used in ASD classification only selects kernel types, namely: linear.

Based on the experiments conducted in this study, Va as a feature selection method has a disadvantage, namely: it has not been optimal in obtaining relevant features for the ASD classification process. Whereas C4.5 and Ripper [10] have weaknesses when the dataset is not balanced. For the classification algorithm used in this study, namely: SVM, RF, and ANN has advantages in terms of classification of datasets that are not balanced. On the other hand, ANN has a weakness in classifying small datasets and computing time is quite long.

\section{Conclusion}

This study used the feature Va selection method and three machine learning algorithms for the classification of 3 datasets (AQ-child, AQ-adolescent, AQ-adult): SVM, RF, and ANN. The experimental results show that the three classifiers could increase the level of sensitivity and specificity in previous study [10]. Specifically, the comparison with previous study [10] on AQ-Adult using RF succeeded in producing a higher sensitivity of $87.89 \%$ while the level of specificity of AQ-Adolescent could be increased using Linear SVM of $86.33 \%$. For further research, the performance of the classification can still be improved using a classifier and other feature selection methods.

\section{References}

1. H. V Ratajczak, Theoretical aspects of autism: Causes - A review, J. Immunotoxicol. 8 (2011) 68-79.

2. C. Plauche and P. Care, Identification and evaluation of children with autism spectrum, Am. Acad. Pediat. 120 (2007) 1183-1215.

3. C. Lord, S. Risi, P. S. DiLavore, C. Shulman, A. Thurm, and A. Pickles, Autism from 2 to 9 years of age, Arch. Gen. Psychiatry. 63 (2006) 694-701.

4. CDC, Screening and Diagnosis of Autism Spectrum Disorder. [Online]. Available: https://www.cdc.gov/ncbddd/autism/screening.html. [Accessed: 10-Apr-2019].

5. C. W. Rishel, C. Greeno, S. C. Marcus, M. K. Shear, and C. Anderson, Use of the child behavior checklist as a diagnostic screening tool in community mental health, Res. Soc. Work Pract. 15 (2005) 195-203.

6. P. A. DA Krug, JR Arick, Autism screening instrument for educational planning-ASIEP 2, J. Psychoeduc. Assess. 16 (1998) 280-285.

7. C. Allison, B. Auyeung, and S. Baron-cohen, Toward brief ' red flags' for autism screening: the short autism spectrum quotient and the short quantitative, J. Am. Acad. Child. Adolesc. Psychiatry. 51 (2012) 202212.e7.

8. National Institute for Health and Care Excellence, Autism spectrum disorder in adults: diagnosis and management, 2012. [Online]. Available: https://www.nice.org.uk/guidance/cg142.

9. Autism Research Centre, Downloadable Tests, 2012. [Online]. Available: https://www.autismresearchcentre.com/arc_tests

10. F. Thabtah, F. Kamalov, and K. Rajab, A new computational intelligence approach to detect autistic features for autism screening, International J. Med. Inf. 117 (2018) 112-124.

11. J. A. Kosmicki, V. Sochat, M. Duda, and D. P. Wall, Searching for a minimal set of behaviors for autism detection through feature selectionbased machine learning, Trans. Psychiat. 5 (2015) e514.

12. M. J. Maenner, M. Yeargin-Allsopp, K. N. Van Braun, D. L. Christensen, 
and L. A. Schieve, Development of a machine learning algorithm for the surveillance of autism spectrum disorder, PLoS One. 11 (2016) 1-11.

13. F. Zhang, P. Savadjiev, W. Cai, Y. Song, Y. Rathi, B. Tunç, D. Parker, T. Kapur, R. T. Schultz, N. Makris, R. Verma, and L. J. O'Donnell, Whole brain white matter connectivity analysis using machine learning: An application to autism, Neurolmage, 172 (2017) 826-837.

14. N. A. Almansour, H. F. Syed, N. R. Khayat, R. K. Altheeb, R. E. Juri, J. Alhiyafi, S. Alrashed, and S. O. Olatunji, Neural network and support vector machine for the prediction of chronic kidney disease: A comparative study, Comp. Biol. Med. 109 (2019) 101-111.

15. C. C. Wu, W. C. Yeh, W. D. Hsu, M. M. Islam, P. A. (Alex) Nguyen, T. N. Poly, Y. C. Wang, H. C. Yang, and Y. C. (Jack) Li, Prediction of fatty liver disease using machine learning algorithms, Comp. Biol. Med. 170 (2019) 23-29.

16. F. Thabtah, Autistic Spectrum Disorder Screening Data for Children $\begin{array}{llll}\text { Data } & \text { Set, } & 2017 & \text {.[Online] }\end{array}$ http://archive.ics.uci.edu/ml/datasets/Autistic+Spectrum+Disorder+Scree ning+Data+for+Children++. [Accessed: 10-Apr-2019].

17. F. Thabtah, Autistic Spectrum Disorder Screening Data for Adolescent Data Set, 2017. [Online]. Available:
https://archive.ics.uci.edu/ml/datasets/Autistic+Spectrum+Disorder+Scre ening+Data+for+Adolescent+++. [Accessed: 10-Apr-2019].

18. F. Thabtah, Autistic Spectrum Disorder Screening Data for Adult Data Set, 2017. [Online]. Available: https://archive.ics.uci.edu/ml/datasets/Autism+Screening+Adult. [Accessed: 10-Apr-2019].

19. D. McSherry. Strategic induction of decision trees bt - research and development in expert systems xv. Res. Dev. Expert Syst. XV, 1999.

20. Huan Liu and R. Setiono, Chi2: feature selection and discretization of numeric attributes, IEEE $7^{\text {th }}$ Int. Conferene on Tools with Artificial Intelligence, 1995, pp. 388-391.

21. Y. Tang and J. Zhou, The performance of PSO-SVM in inflation forecasting, 12 th Int. Conf. Serv. Syst. Serv. Manag. ICSSSM, 2015, pp. $1-4$.

22. L. Breiman, Random forests, J. Mach. Learn. 45 (2001) 5-32.

23. I. Witten and E. Frank. Data mining $2^{\text {nd }}$ edition: Practical machine learning tools and techniques. US of America: Morgan Kaufmann. 2005.

24. Scikit-learn Machine Learning in Python. [Online]. Available: https://scikit-learn.org/stable/. [Accessed: 10-Apr-2019]. 